\title{
Threat and error management in terms of coordination between crew and ATC team based on Bayesian method
}

\author{
Xiaolong Zhu ${ }^{1, a,{ }^{*}, \text { Haitian Zhang }}{ }^{2, \mathrm{~b}}$ and Zhiqin $\mathrm{Huo}^{3, \mathrm{c}}$ \\ ${ }^{1}$ School of Physics and Information Engineering, Jianghan University, Wuhan 430056, Hubei, \\ China \\ ${ }^{2}$ Hubei Air Traffic Management Sub-Bureau of CAAC, Wuhan 430302, Hubei, China \\ ${ }^{3}$ China Academy of Civil Aviation Science and Technology, Beijing 100028, China \\ axlzhu@jhun.edu.cn, bzhanghaitian1220@163.com, chuozhq@mail.castc.org.cn \\ ${ }^{*}$ Corresponding author
}

Keywords: TEM model, Coordination Between Crew and ATC, Bayesian Evaluation, Correlation.

\begin{abstract}
A new TEM model is proposed on the basis of coordination between crew and ATC team and an analysis of the sample data about threat, error and undesired state is conducted by using Bayesian model. The analysis obtains the correlations of the three kinds of safety information above through studying the respective posterior probabilities of threats and errors under unexpected state. Investigation on 785 sample data shows that the three kinds of threats most correlated to unexpected state are controllers' error to pilots, pilots' error to controllers, and air traffic flow threat; and that the three kinds of errors most correlated to unexpected state are controllers' error of speaking and listening, releasing the mistake order by controllers' wrong decision, and pilots' error operation of A/C. Therefore, the safety control departments of airlines and ATC units should lay their emphasis on human error, especially on the communication errors between crew and ATC team.
\end{abstract}

\section{面向空地配合的威胁与差错管理贝叶斯模型分析}

\author{
朱小龙 ${ }^{1, a,{ }^{*}}$, 张海天 ${ }^{2, b}$, 霍志勤 $3, c$ \\ 1江汉大学物理与信息工程学院, 武汉, 湖北, 中国 \\ 2民航湖北空管分局, 武汉, 湖北, 中国 \\ 3中国民航科学技术研究院, 北京, 中国 \\ axlzhu@jhun.edu.cn, bzhanghaitian1220@163.com, chuozhq@mail.castc.org.cn \\ 通讯作者
}

关键词: TEM模型；空地配合；贝叶斯估计；相关性

中文摘要. 构建面向空管班组与飞行机组配合的威胁与差错管理模型, 并针对收集到的数据 样本进行贝叶斯模型分析。通过研究非期望状况发生条件下威胁与差错各自发生的后验概率, 确定三者之间的紧密联系程度。针对收集到的785起数据样本进行了研究, 结果表明: 与空中 非期望状况的发生相关性最大的3类威胁依次是管制员差错对飞行员造成的威胁，飞行员差错 对管制员造成的威胁，空中交通流量分布; 与空中非期望状况的发生相关性最大的 3 类差错依 次是管制员口误、误听，管制员决策发布错误的管制指令，飞行员操作航空器高度、航向、 速度偏差。航空公司和空管部门应制定相应的程序重点管控人为差错, 特别是空地之间的通 讯差错。 


\section{1. 引言}

威胁与差错管理（TEM）是一种包含了航空运行和人的表现的安全概念, 帮助我们从运 行的角度来理解在动态和复杂的运行环境中安全和人的表现之间的内部关系。国际民航界已 将TEM分析框架运用于航线运行安全审计（LOSA）、机组资源管理（CRM）、空管正常运 行安全监测（NOSS）和空管管制员培训中。

对空中交通运行过程中的威胁、差错及非期望状况的归类、统计和数据分析是当前民航 研究的重要前沿领域。国内外研究人员已分别针对航空公司、空管等不同层面及部门提出了 相应的TEM模型。霍志勤等 ${ }^{[1]}$ 分别界定了民用航空领域威胁、差错、非期望状况的概念, 丰 富了威胁与差错管理的框架形式; 张晓全 ${ }^{[2]}$ 提出了基于CRM行为的飞行运行威胁与差错管理 模型; 何煦 ${ }^{[3]}$ 分析了空管运行中面临的威胁和可能出现的差错, 并提出了管理威胁与差错的 策略和措施; 石凯 ${ }^{[4]}$ 从威胁与差错管理理论出发, 对我军运输机实际飞行的机组资源管理过 程进行了分析, 提出威胁与管理对策以及机组人员能力需求; Anthony ${ }^{[5]}$ 等人利用美国强制报 告系统数据, 采用TEM 框架研究地面运行、空域运行与空管因素导致的事故/事故征候之间 的关系。以上研究注重TEM概念的引入与分类, 但缺乏相关定量的分析与评价。目前已有学 者对TEM的定量技术方面进行了探索，采用归类分析、概率统计、网络层次分析法、贝叶斯 模型和贝叶斯网络模型研究各项因素之间的相关性。霍志勤等 ${ }^{[6]}$ 对跑道侵入不安全事件中的 威胁、差错等数据进行定量研究, 识别不同类别数据的相关性, 并尝试对结果和影响因素进 行多元回归分析。吕人力等 ${ }^{77]}$ 对民航空管各类安全信息的 TEM分布特征进行比较研究。杨虎 等 ${ }^{[8]}$ 构建了MPL培训中TEM能力的评价指标体系, 并采用网络层次分析与模糊综合评价相结 合的方法对某飞行训练机构的 TEM 能力进行了评价。Dodd B. ${ }^{[9]}$ 在处理安全数据时首次引入贝 叶斯模型, 并阐述了如何利用这些数据实现风险管理; 曹海峰等 ${ }^{[10,11]}$ 首次利用贝叶斯和贝叶 斯网络模型针对空管领域的威胁与差错事件进行量化分析, 对一定情境条件下特定人误类型 的后验概率进行了风险量化研究, 提供了基于威胁及差错发生的先验概率, 获得意外状态与 各类威胁及差错间关系的后验概率的计算方法, 并对威胁、差错及非期望状况的相关性大小 进行排序。

作为航空器运行阶段的安全、效率与空中秩序的共同保障者，管制员与飞行员之间必须 密切协作与配合。空地配合不当及航空器故障等导致的空中交通运行不正常情况（包括不安 全事件) 已经达到事件总数的一半以上。虽然目前分别针对这两者的威胁与差错管理研究已 经具备一定的基础, 但都是将飞行机组和管制班组作为独立团队看待, 强调的都是单一工种 内部的协作与配合以及对各自子系统可靠性的影响。尚未将两者作为一个整体纳入到威胁与 差错管理框架当中, 忽视了空地之间的交互作用。与此同时, 空中交通运行保障压力剧增, 迫切需要更加紧密的空地协作配合运行模式保障空中交通安全。因此, 构建面向空管班组与 飞行机组配合的威胁与差错管理框架, 对于提高空地配合质量, 提升航空安全水平和运行效 率, 以及高效维持空中飞行秩序具有积极意义。

本文将从飞行员与管制员的双视角讨论威胁与差错管理问题，建立面向空地配合的TEM 模型, 界定空地配合中的威胁、差错及非期望状况, 并针对收集到的数据样本进行了贝叶斯 模型分析，从而确定与空中交通非期望状况的发生联系紧密的威胁和差错类型。

\section{2. 面向空地配合的TEM模型分析}

作为航空器运行阶段双主体的飞行员、管制员虽然能够在一定程度上通过机组资源管理 (CRM) 和班组资源管理 (TRM) 分别对各自的工作任务进行有效管理, 但对于复杂的空中 
表1 威胁、差错及非期望状况类型统计

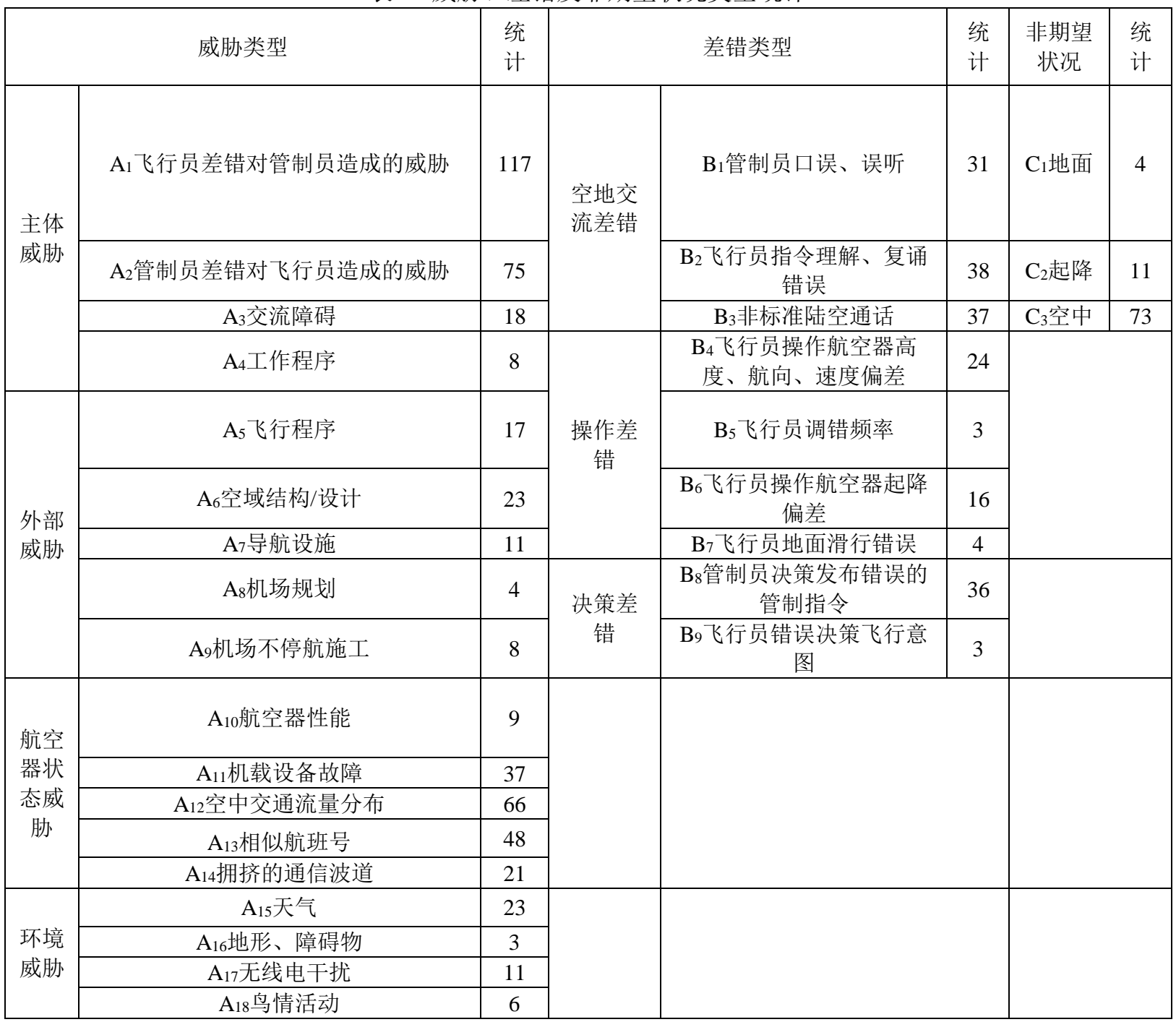

交通运行而言，双方往往需要管理共同的或者由对方差错造成的威胁，降低外在威胁和 对方差错造成的影响, 协同处置空中交通非期望状况, 以确保单一客体一一空中交通运行的 安全。飞行员与管制员有着共同的安全期望，而各自具体的安全目标却有所不同。飞行员重 点关注单架航空器的运行状态, 管制员侧重于保障空中交通整体运行态势的安全顺畅, 但彼 此同样需要对方的全力协助与配合。图（1）所示为基于空地配合的威胁与差错管理模型。与 单一主体的TEM模型相比，基于空地协作新模式的TEM模型应重点强调空地之间的相互影响 以及作为双主体的飞行员与管制员对于威胁、差错与非期望状况的协同管理。 


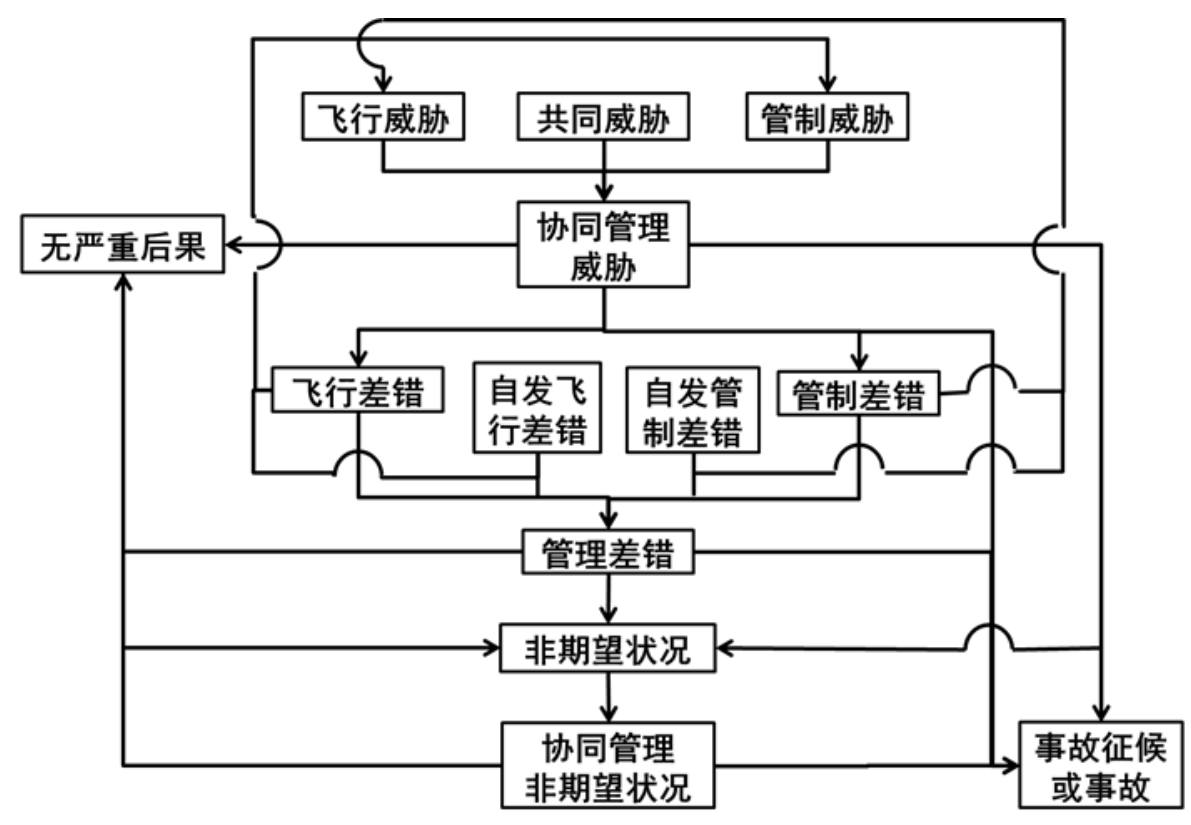

图1 基于空地配合的TEM模型

参考国际民航组织对空管TEM的分类标准，同时结合空地配合的实际情况，将威胁、差 错以及非期望状况进行界定并分为以下若干大类和子类，见表1。

\section{1 需要空地配合管理的空中交通运行中的威胁界定}

需要空地配合进行协同管理的威胁是指：空中交通运行过程中，在飞行员或管制员控制 能力之外的事件或差错, 影响空地任何一方, 它增加了运行的复杂性, 提高了运行难度, 但 必须设法并且需要通过空地配合将其保持在可接受安全水平之内。如果不管理或者错误管理 威胁，可能导致差错甚至进入非期望状况。将飞行员和管制员作为一个整体对待，根据影响 空中交通安全运行的四个因素: 人员操控、外部设施、航空器状态及外界环境, 可以把需要 空地配合协同管理的威胁分为:（1）主体威胁;（2）外部威胁;（3）航空器状态威胁; 环境威胁。

\section{2 需要空地配合管理的空中交通运行中的差错界定}

需要空地配合进行协同管理的威胁是指：空中交通运行过程中，在飞行员或管制员控制 能力之外的事件或差错, 影响空地任何一方, 它增加了运行的复杂性, 提高了运行难度, 但 必须设法并且需要通过空地配合将其保持在可接受安全水平之内。如果不管理或者错误管理 威胁, 可能导致差错甚至进入非期望状况。将飞行员和管制员作为一个整体对待, 根据影响 空中交通安全运行的四个因素: 人员操控、外部设施、航空器状态及外界环境, 可以把需要 空地配合协同管理的威胁分为:（1）主体威胁;（2）外部威胁;（3）航空器状态威胁; 环境威胁。

\section{3 需要空地配合管理的空中交通运行中的非期望状况界定}

当空中交通运行状态已经偏离了预期的正常状况进入危险的状况时，单架航空器或者航 空器之间的运行状态产生了实际的偏差, 即构成空中交通运行中的非期望状况。不论是飞行 员还是管制员，如果之前未能有效地管理威胁和差错，都可能导致非期望状况的出现。非期 望状况是一个瞬间、快速发展的过程, 管理非期望状况往往比管理威胁和差错更为紧迫, 也 是空地双方防止发生事故征候或事故的最后机会。按照航空器运行的不同阶段, 可以将非期 望状况分为: 地面非期望状况、起降非期望状况和空中非期望状况。非期望状况已经开始牺 牲安全裕度, 需要空地双方及时共同采取措施, 控制事态进一步恶化。 


\section{3. 空地配合的贝叶斯模型分析研究}

\section{1 贝叶斯模型}

作为对民航安全管理TEM模型的数据信息进行相关性研究的有效方法一一贝叶斯估计方 法，可以用于研究空地配合的威胁差错与非期望状况的相关性分析。

设事件 $A_{\mathrm{i}}(\mathrm{i}=1, \cdots, \mathrm{n})$ 为相互独立事件，若 $\mathrm{C}$ 为已知结果，则 $\mathrm{C}$ 由事件 $\mathrm{A}_{\mathrm{i}}$ 引发的概率为

$$
\mathrm{P}\left(\mathrm{A}_{\mathrm{i}} \mid \mathrm{C}\right)=\frac{\mathrm{P}\left(\mathrm{C} \mid \mathrm{A}_{\mathrm{i}}\right) \mathrm{P}\left(\mathrm{A}_{\mathrm{i}}\right)}{\Sigma_{\mathrm{j}}^{\mathrm{p}} \mathrm{P}\left(\mathrm{C} \mid \mathrm{A}_{\mathrm{i}}\right) \mathrm{P}\left(\mathrm{A}_{\mathrm{i}}\right)}
$$

其中, $P\left(A_{i} \mid C\right)$ 表示当结果 $C$ 发生时事件 $A_{i}$ 发生的概率。当已知 $P(C)$ 时, 可以简化为

$$
\mathrm{P}\left(\mathrm{A}_{\mathrm{i}} \mid \mathrm{C}\right)=\frac{\mathrm{P}\left(\mathrm{C} \mid \mathrm{A}_{\mathrm{i}}\right) \mathrm{P}\left(\mathrm{A}_{\mathrm{i}}\right)}{\mathrm{P}(\mathrm{C})}
$$

根据 $\mathrm{C}$ 结果下 $\mathrm{A}_{\mathrm{i}}$ 发生的后验概率，可间接说明事件 $\mathrm{A}_{\mathrm{i}}$ 与结果 $\mathrm{C}$ 之间的相关性。

\section{2 空地配合的威胁、差错、非期望状况类型统计}

通过不正常情况调查报告、安全运行质量监督检查和员工自愿报告3种途径收集到某ATC 单位的785起与空地配合有关联的威胁、差错及非期望状况数据信息（2008年至今）进行统计 分类, 得到的结果如表 1 所示。其中, $A_{i}$ 代表第 $i$ 类威胁 $(i=1, \ldots, 18), B_{j}$ 代表第 $j$ 类差错 $(j=1, \ldots, 9)$, $\mathrm{C}_{\mathrm{k}}$ 代表第 $\mathrm{k}$ 类非期望状况 $(\mathrm{k}=1, \ldots, 3)$ 。从统计结果可以看出, 各类威胁中发生次数最多的是 主体威胁中飞行员差错对管制员造成的威胁，主要表现为飞行员复诵指令错误、陆空通话不 规范、航空器误操作等, 导致管制员被动应对, 从而诱发管制员差错; 其次是管制员差错对 飞行员造成的威胁, 主要表现为管制员呼错航班号, 非标准通话, 发布错误指令等, 从而导 致飞行员执行错误的管制指令; 航空器状态威胁中的空中交通流量分布和相似航班号两项因 素发生的频率也较高, 单管制扇区飞行流量超负荷、相似航班号出现概率增大对管制员决策、 管制员和飞行员的监听复诵都产生负面影响。各类差错中发生次数排在前三位的分别是空地 交流差错中的飞行员指令理解、复诵错误, 非标准陆空通话, 以及决策差错中管制员决策发 布错误的管制指令。这些差错可以因其他威胁诱导发生也可以主体双方自发产生。非期望状 况中则是空中非期望状况次数最多, 这与研究对象的运行特点密切相关, 也说明空中意外状 态应当成为空地配合TEM研究的重点内容。

从上表中虽然能够看出各类威胁、差错和非期望状况的分布，但没有显示出非期望状况 发生时各类威胁与差错类型出现的频率, 因此无法获得各类因素之间的相关性。因此, 在上 述TEM数据统计的基础上, 需要分别对威胁与非期望状况, 差错与非期望状况应用贝叶斯模 型进行数据相关性的计算研究，以获取非期望状况发生时威胁、差错同时发生的后验概率。

\section{3 空地配合的相关性计算与分析}

\begin{tabular}{|c|c|c|c|c|}
\hline 威胁/差错类型 & 次数 & 威胁\&非期望状况 & $\begin{array}{l}\text { 次 } \\
\text { 数 }\end{array}$ & $\mathrm{P}\left(\mathrm{A}_{\mathrm{i}} \mid \mathrm{C}_{2}\right) / \mathrm{P}\left(\mathrm{B}_{\mathrm{i}} \mid \mathrm{C}_{2}\right)$ \\
\hline $\begin{array}{c}\mathrm{A}_{1} \text { 飞行员差错对管制员 } \\
\text { 造成的威胁 }\end{array}$ & 117 & $\begin{array}{c}\mathrm{A}_{1} \text { 飞行员差错对管制员造成的威 } \\
\text { 胁 } \& C_{3} \text { 空中 }\end{array}$ & 35 & 0.4795 \\
\hline $\begin{array}{c}\mathrm{A}_{2} \text { 管制员差错对飞行员 } \\
\text { 造成的威胁 }\end{array}$ & 75 & $\begin{array}{c}\mathrm{A} 2 \text { 管制员差错对飞行员造成的威 } \\
\text { 胁 } \& C_{3} \text { 空中 }\end{array}$ & 46 & 0.6301 \\
\hline $\mathrm{A}_{11}$ 机载设备故障 & 37 & $\mathrm{~A}_{11}$ 机载设备故障\& $\mathrm{C}_{3}$ 空中 & 8 & 0.1096 \\
\hline $\mathrm{A}_{12}$ 空中交通流量分布 & 66 & $\mathrm{~A}_{12}$ 空中交通流量分布 $\& \mathrm{C}_{3}$ 空中 & 28 & 0.3836 \\
\hline $\mathrm{A}_{13}$ 相似航班号 & 48 & $\mathrm{~A}_{13}$ 相似航班号 \& $\mathrm{C}_{3}$ 空中 & 17 & 0.2329 \\
\hline $\mathrm{B}_{1}$ 管制员口误、误听 & 31 & $\mathrm{~B}_{1}$ 管制员口误、误听 \& $\mathrm{C}_{3}$ 空中 & 21 & 0.2877 \\
\hline $\begin{array}{c}\mathrm{B}_{2} \text { 飞行员指令理解、复诵 } \\
\text { 错误 } \\
\end{array}$ & 38 & $\begin{array}{c}\mathrm{B}_{2} \text { 飞行员指令理解、复诵错误 } \& \\
\mathrm{C}_{3} \text { 空中 }\end{array}$ & 16 & 0.2192 \\
\hline $\mathrm{B}_{3}$ 非标准通话用语 & 37 & $\mathrm{~B}_{3}$ 非标准通话用语 $\& \mathrm{C}_{3}$ 空中 & 5 & 0.0685 \\
\hline
\end{tabular}

表2 威胁、差错与非期望状况的贝叶斯估计 
续表2

\begin{tabular}{|c|c|c|c|c|}
\hline $\begin{array}{c}\mathrm{B} 4 \text { 飞行员操作航空器高 } \\
\text { 度、航向、速度偏差 }\end{array}$ & 24 & $\begin{array}{c}\mathrm{B}_{4} \text { 飞行员操作航空器高度、航向、 } \\
\text { 速度偏差 } \mathrm{C}_{3} \text { 空中 }\end{array}$ & 17 & 0.2329 \\
\hline $\begin{array}{c}\mathrm{B}_{8} \text { 管制员决策发布错误 } \\
\text { 的管制指令 }\end{array}$ & 36 & $\begin{array}{c}\mathrm{B}_{8} \text { 管制员决策发布错误的管制指 } \\
\text { 令 \& } \mathrm{C}_{3} \text { 空中 }\end{array}$ & 21 & 0.2877 \\
\hline
\end{tabular}

通过贝叶斯估计，可以计算出在空中非期望状况发生时，哪种威胁或者差错同时发生的 比例最高, 并由此建立起风险控制模型。针对统计概率排在前五位的威胁、差错与空中非期 望状况进行贝叶斯估计计算, 计算结果见表 2 。以航空器状态威胁中相似航班号 $A_{13}$ 与空中意 外状态 $C_{3}$ 为例, 计算出 $P\left(A_{13} \mid C_{3}\right)$ 的概率, 即当空中交通非期望状况发生时相似航班号威胁发生 的概率, 由此可以间接说明相似航班号威胁与空中非期望状况之间的相关性。计算步骤为: 从表1不正常情况调查报告、安全运行质量监督检查和员工自愿报告3种途径收集到的785起与 空地配合有关联的威胁、差错及非期望状况数据中, 统计出相似航班号威胁 $A_{13}$ 发生的概率为 $(48 / 785)$, 空中意外状态 $C_{3}$ 发生的概率为 $(73 / 785)$; 从表 2 统计数据可知, 相似航班号威胁 $A_{13}$ 发 生且导致空中意外状态 $C_{3}$ 同时发生的次数为 17 , 因此根据公式(2)可以得到 $C_{3}$ 条件下 $A_{13}$ 发生的 后验概率为

$$
\mathrm{P}\left(\mathrm{A}_{13} \mid C_{3}\right)=\mathrm{P}\left(C_{3} \mid A_{13}\right) \times \frac{P\left(A_{13}\right)}{P\left(C_{3}\right)}=23.29 \%
$$

这表示当空中交通非期望状况发生时，出现相似航班号威胁 $A_{13}$ 的概率为 $23.29 \%$

根据表 2 的统计与计算结果表明, 空中非期望状况的发生同威胁差错的发生概率并不成正 比。有些威胁、差错因素本身发生的频率较高, 但并不容易导致非期望状况的发生。例如, 飞行员差错对管制员造成的威胁 $A_{1}$ 发生次数为 117 次, 管制员差错对飞行员造成的威胁 $A_{2}$ 发生 的次数为 75 次, 两者发生的频率比为 1.56 。但是, 根据贝叶斯估计, 飞行员差错对管制员造 成的威胁与空中非期望状况的相关性 $A_{1} \& C_{3}$ 为 0.4795 , 低于管制员差错对飞行员造成的威胁与 与空中非期望状况的相关性 $A_{2} \& C_{3}$ 为 0.6301 。这说明, 在空中非期望状况 $C_{3}$ 发生的情况下, 管 制员差错对飞行员造成的威胁发生非期望状况的可能性更大。因此, 作为对风险的管控, 相 对而言更应当加强对管制员差错造成的威胁的防范。在空地配合中的差错方面, 管制员口误、 误听 $B_{1}$ 发生次数为 31 次, 小于非标准通话用语 $B_{2}$ 发生的次数 37 次, 但是, 管制员口误、误听 $B_{1}$ 与空中非期望状况 $C_{3}$ 的相关性为 0.2877 , 明显大于非标准通话用语 $B_{3}$ 与空中非期望状况 $C_{3}$ 的相 关性 0.0685 , 这说明, 在空中非期望状况发生的情况下, 管制员口误、误听差错发生的可能 性比非标准通话用语差错发生的可能性更大。在平时的空中交通运行中, 虽然空地双方存在 大量的非标准通话用语, 但并不容易造成双方的误解; 而一方的口误或误听指令相对而言后 果更严重, 往往会直接导致飞行冲突。为了有效防止空中非期望状况的发生, 建议管制员加 强对机组复诵的监听, 特别注重相似航班号、英语指令的发布与监听; 对机组复诵错误要及 时发现并更正; 对于机组复诵有疑问时, 及时核实, 确保机组收到正确指令; 主副班管制员 要加强对飞行员执行指令情况的监控; 关于高度、航向、速度、频率等关键指令管制员要分 段发布, 防止机组误听、漏听。由此可见, 不能简单的根据威胁和差错的发生概率来判定其 重要性, 而要根据它们与非期望状况的相关性来判断。

为便于直观比较, 下面分别用图 2 和图3来表示空中非期望状况与威胁、差错的相关程度。

从图 2 中可知, 空中非期望状况与各类威胁的相关性从大到小依次为管制员差错对飞行员 造成的威胁、飞行员差错对管制员造成的威胁、空中交通流量分布、相似航班号、机载设备 故障。其中, 空地双方由于自身错误给对方造成的威胁远高于其他类型的威胁。而这些威胁 往往可以通过空地双方相互配合进行有效管理, 阻止态势进一步发展为空中非期望状况。空 管单位和航空公司可以采取以下措施提高飞行员与管制员之间的配合水平, 例如: 加强空地 人员的跨行业培训、交流, 了解双方容易出现差错的环节, 增强自身素质与协作能力; 重新 编排和优化更加吻合的空地配合程序; 加强安全信息的共享与分析等。 


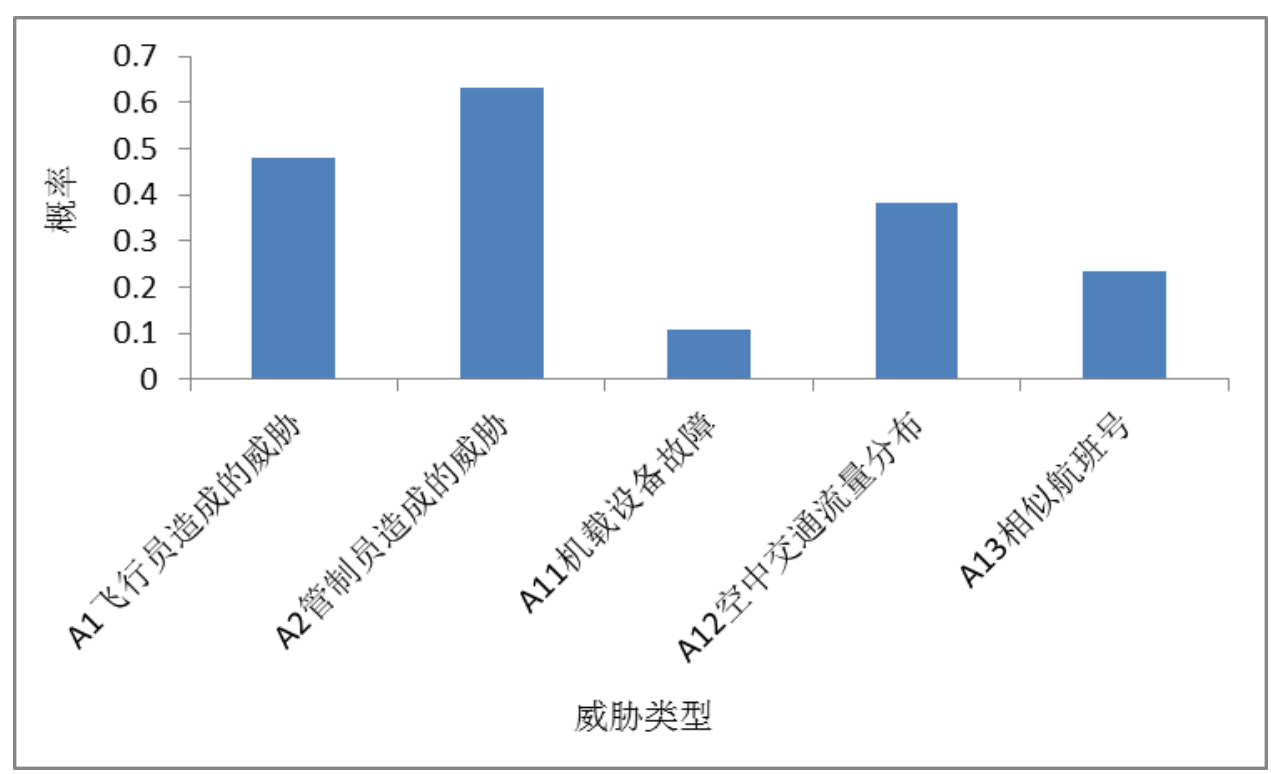

图2 空中非期望状况与各类威胁的相关性

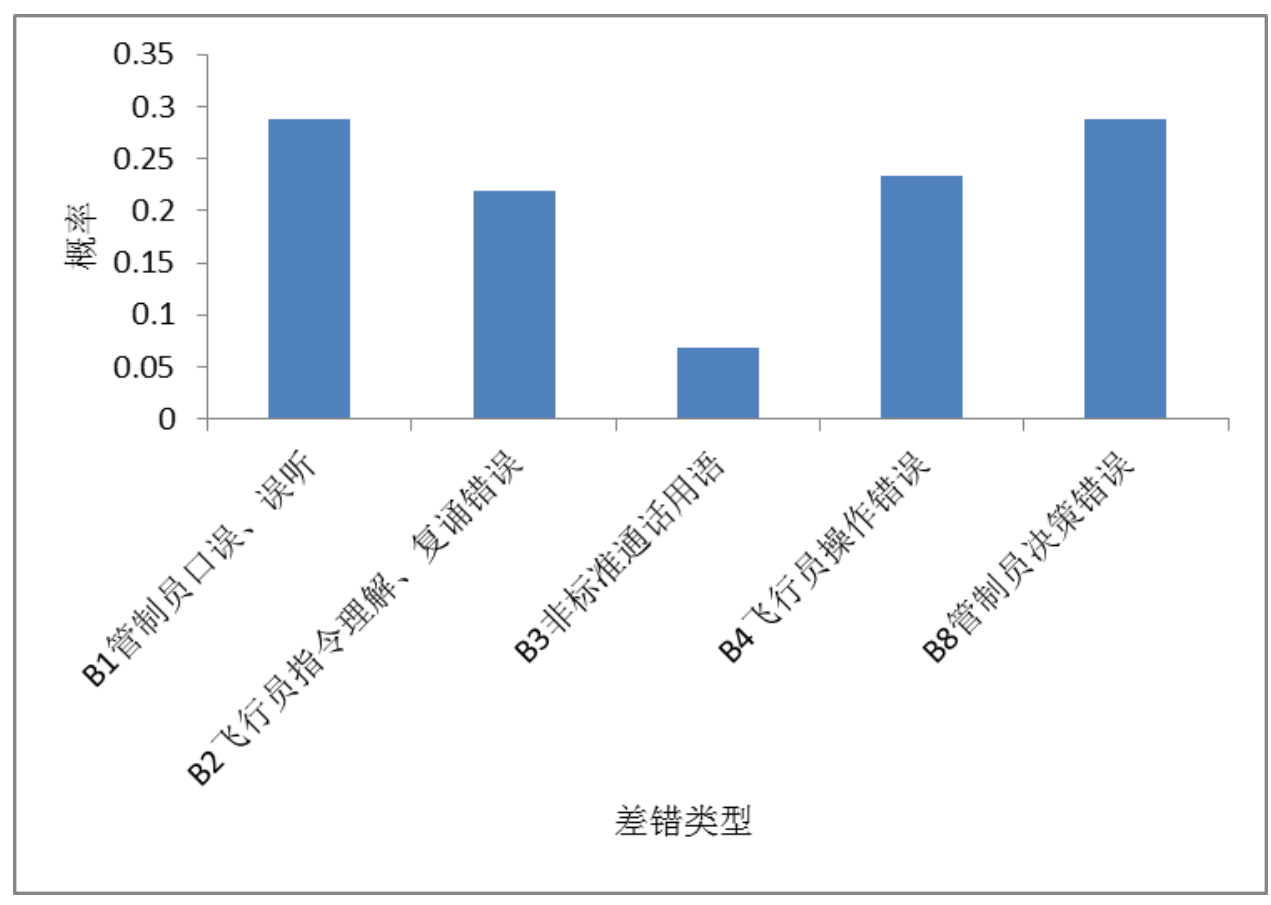

图3 空中非期望状况与各类差错的相关性

从图3中可知，空中非期望状况与各类差错的相关性从大到小依次为管制员决策发布错 误的管制指令, 管制员口误、误听, 飞行员操作航空器高度、航向、速度偏差, 飞行员指令 理解、复诵错误，非标准通话用语。管制员口误、误听 $B_{1}$ 和管制员决策错误 $B_{8}$ 发生的条件概 率相同, 但前者发生的频率要低于后者。这说明管制员发生口误、误听往往更容易直接导致 航空器飞行冲突的发生。所以, 尽管管制员口误、误听本身出现的概率相对较低, 但其发生 后可能导致的后果相对要严重的多。

这些数据都提示航空公司和空管部门的管理者应将防范的重点放在与空中非期望状况相 关性较高的威胁与差错上, 通过各种管理手段改善空地配合水平，从而提高对威胁与差错的 管理效率。例如管制员口误、误听差错造成的空中态势恶化的可能性较大，空管部门应当在 运行过程中采取有效措施重点防范此类差错的发生。 


\section{4. 结束语}

本文建立了面向空地配合的TEM模型，对空地双视角下的威胁、差错和非期望状况进行 分类。通过对非期望状况使用贝叶斯估计方法，基于威胁、差错及非期望状况发生的先验概 率计算出与非期望状况相关的各类威胁与差错的后验概率, 找出与非期望状况联系紧密的威 胁、差错类型, 为航空公司和空管部门应制定相应的风险管控措施提供科学依据。在接下来 的研究中, 可以通过贝叶斯网络模型分析威胁、差错和非期望状况之间的网络拓扑结构, 对 非期望状况与威胁、差错的相关性做进一步描述。

\section{致谢}

本文为国家自科基金民航联合基金项目《基于复杂网络理论的国家航路网络宏观结构评 估与优化》(U1433121)和湖北省教育厅科学研究计划项目《黏菌生长模拟建立高效运输网络 的研究》(B2014065)的阶段性成果之一。

\section{References}

[1] Z.Q. Huo, R.L. Lv and Y.J. Shi, Threat and error management in civil aviation operation, Safety Science Journal, vol.17, pp. 61-65, 2007.

[2] X.Q. Zhang, Threat and error management in flight operation based on CRM, International Symposium on Safety Science and Technology, conference proceedings, pp. 373-376, 2008.

[3] X. He, Analysis of threats and errors in the operation of air traffic control, Journal of Civil Aviation Flight University of China, vol. 19, pp. 15-19, 2008.

[4] K. Shi, S.D. Wei, K.J. Zhang and J.J. Zhou, The crew resource management theory and application based on TEM, Value Engineering, vol. 11, pp. 19-21, 2012.

[5] M.P. Anthony, A.W. Douglas, S. Scott, Air traffic control related accidents and incidents: a human factors analysis, The 11th International Symposium on Aviation Psychology, Columbus, OH, pp. 1-4, 2001.

[6] Z.Q. Huo, S.C. Han, Analysis on the threats and errors caused by the runway incursions and the corresponding controlling ways, Journal of Safety and Environment, vol.12, pp. 192-196, 2012.

[7] R.L. Lv, H.F. Cao, Y. Zhou and F.L. Bai, Comparative study of three sources for safety information in civil aviation, Journal of Safety and Environment, vol.10, pp. 170-174, 2010.

[8] H. Yang, L.S. Pan and Y. You, Evaluation of threat and error management capability in MPL training based on ANP-Fuzzy, Safety and Environmental Engineering, vol.5, pp. 80-85, 2014.

[9] B. Dodd, Safety data integration can lead to better understanding of risk, International Civil Aviation Organization journal, vol.62, pp. 21-22, 2007.

[10]H.F. Cao and T. Li, Study on Threat, Error and Undesired States in Air Traffic Control Based on Bayesian Method, China Safety Science Journal, vol.21, pp. 85-89, 2011.

[11] H.F. Cao, F.L. Bai and T. Li, On the correlation among threats, errors, and undesired states in air traffic control based on a Bayesian network model, Journal of Safety and Environment, vol.13, pp. 188-191, 2013. 